\title{
Von der Kontrastmittelsonografie bis zum Einsatz im OP: Ultraschall ist ein wichtiges und vielseitig einsetzbares bildgebendes Verfahren in der Medizin
}

Leipzig - Ob in Diagnostik, Intervention oder Therapie - der Ultraschall ist längst ein unverzichtbarer Bestandteil in allen medizinischen Disziplinen und unterliegt einer ständigen Weiterentwicklung. Über modernste und zukunftsweisende Techniken diskutierten rund 1700 internationale Experten vom 16. bis 19. Oktober 2019 in Leipzig beim Ultraschallkongress der Deutschen, Österreichischen und Schweizer Gesellschaft für Ultraschall in der Medizin (DEGUM, ÖGUM und SGUM). Auch die Weiterentwicklung des Ultraschalls sowie stetige Fort- und Weiterbildungsmaßnahmen standen im Fokus der Diskussion. Beispiele dafür sind neue und bewährte Einsatzmöglichkeiten der Kontrastmittelsonografie, der Stellenwert von hochauflösenden Ultraschallgeräten, die ein exaktes Operieren ermöglichen, sowie neue gerätetechnische Entwicklungen und Methoden, mit denen Tumoren punktgenau zerstört werden können.

Wird Künstliche Intelligenz (KI) zukünftig den Arzt ersetzen? Der lernende Computer wertet in kurzer Zeit tausende von diagnostischen Bilddaten und Laborwerten aus, stellt eine exakte Diagnose und kann so oft frühzeitiger Krankheiten erkennen als Mediziner. Wie intelligenter Ultraschall die Krebsfrüherkennung revolutioniert, war eines der Highlight-Themen des Dreiländertreffens in Leipzig. „KI kann alle diagnostischen Parameter wie Biomarker, Blutwerte, Risikofaktoren, Bilddaten sowie Ultraschallaufnahmen zusammenfassen und mit großen Datenbanken vergleichen“, erklärte Bernhard Mumm. Der Gastredner hat bei der Kongresseröffnung den Vortrag „Chancen und Risiken von $\mathrm{KI}$ in der Bildgebung“ gehalten. „Jede Krankheit hat eine spezifische Handschrift in diesen Daten“, so Mumm weiter. So könne zum Beispiel eine wesentlich bessere Tumorfrüherkennung durch diese Kombination von vielen diagnostischen Daten erfolgen. Auch die Risiken eines Menschen, eine spezielle Krankheit zu bekommen, könnten frühzeitig entdeckt werden. „Speziell Ultraschall wird sich durch $\mathrm{KI}$ immer mehr von einer reinen qualitativen zu einer quantitativen Bildgebung entwickeln und so eine immer wichtigere diagnostische Rolle spielen, etwa bei der Früherkennung von Herzproblemen und Krebserkrankungen.“

Ultraschall hat auch einen immer höheren Stellenwert im Operationssaal. Beim ultraschallgestützten Operieren hilft Sonografie dem Operateur, das OP-Messer zu führen. „Als Chirurgen operieren wir quasi blind“, erklärte dazu Dr. med. Matthias Wüstner vom Zentrum für Radiologie, Neuroradiologie, Sonografie und Nuklearmedizin am Krankenhaus der Barmherzigen Brüder Trier und Leiter der DEGUM-Sektion Chirurgie. „Wir sehen immer nur die Oberfläche des Organs. Ultraschall bietet die Möglichkeit, unter der Oberfläche zu sehen wie das Messer zu führen ist, um zum Beispiel einen Lebertumor herauszuschneiden, ohne unnötig gesundes Gewebe aus der Umgebung zu entfernen."

Sehr erfolgreich wird das Verfahren auch in der Gynäkologie (Frauenheilkunde) eingesetzt. Professor Dr. med. Markus Hahn, diesjähriger Kongresspräsident des Dreiländertreffens und Leiter der Experimentellen Senologie am Universitätsklinikum Tübingen, ergänzte, dass es für die Prognose der Patientin mit einem Mammakarzinom (Brustkrebs) bei der brusterhaltenden Operation sehr wichtig ist, den Brusttumor komplett zu entfernen, aber gleichzeitig für ihre Lebensqualität genauso bedeutsam, soviel gesundes Gewebevolumen wie möglich in der Brust zu belassen, um ein harmonisches Ergebnis zu erzielen. Es zeigt sich in vielen Studien, dass die Rate an notwendigen Zweiteingriffen durch Einsatz der Sonografie direkt im Operationssaal signifikant reduziert werden kann. Operateure, die diese Präzisionschirurgie anbieten wollen, müssen nicht nur gut operieren können, sondern auch in der Sonografie ausgebildet sein. „Die Hälfte der Brustoperateure der DEGUM setzt das Verfahren schon ein“, sagt Professor Hahn. Das sei das aktuelle Ergebnis einer Umfrage des Arbeitskreises Mammasonografie. Hahns Wunsch ist,

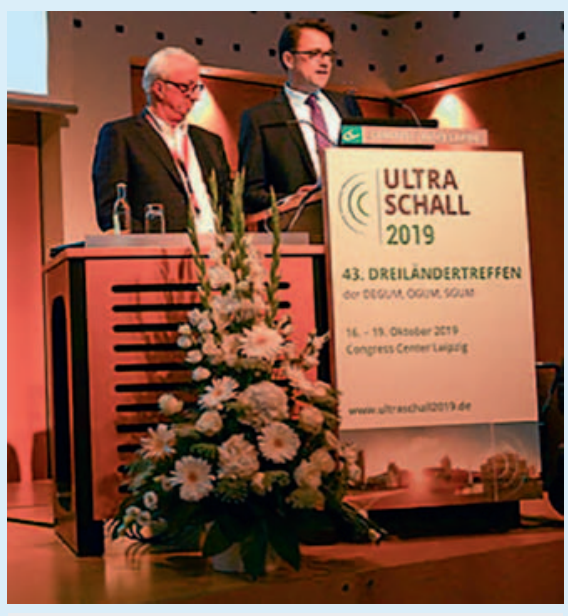

Die Kongresspräsidenten Dr. Hans-Peter Weskott (links) und Prof. Dr. Markus Hahn (rechts). Foto: DEGUM.

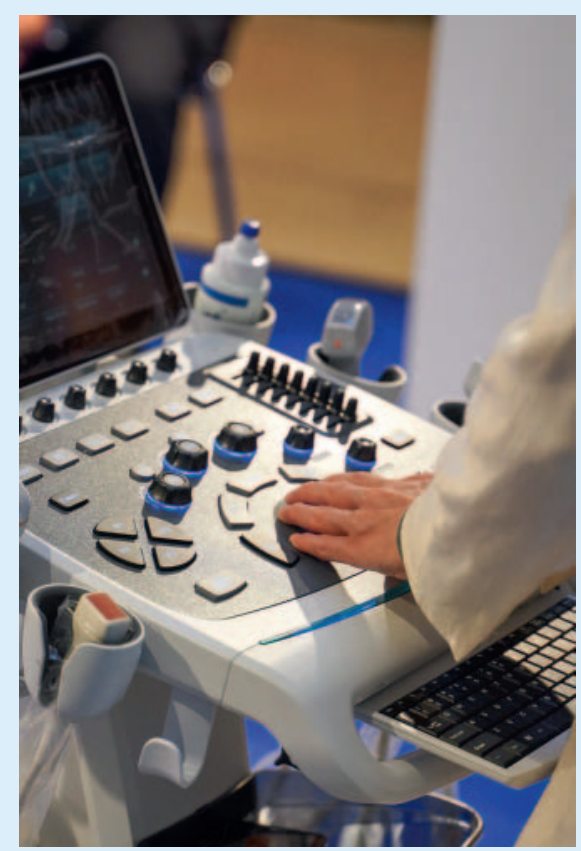

Die Kongressteilnehmer hatten die Möglichkeit an neuesten Geräten zu üben. Foto: DEGUM.

dass diese Ausbildung jedoch flächendeckend von allen ärztlichen Kollegen in Deutschland genutzt wird.

„Wir merken auch, wie das Sonografie-gestützte Verfahren auf mehr und mehr opera- 
tive Fachgebiete übergreift und wir mehr und mehr minimalinvasiv gewebeschonend arbeiten können. Das geschieht natürlich zum Nutzen unserer Patienten. Ebenso profitieren auch Patientinnen, die sich zum Beispiel im Rahmen eines Kinderwunsches aus der Gebärmutter Myome oder Endometrioseherde entfernen lassen müssen und dieses Organ so intakt lassen können“, so Hahn.

Bei der Pressekonferenz des Dreiländertreffens wurde außerdem thematisiert, wie Tumoren per Ultraschall frühzeitig und auf besonders schonende Weise aufgespürt werden können: Mittels Kontrastmittelsonografie können Ärzte oft eher und mit hoher Sicherheit auch im Vergleich mit anderen bildgebenden Verfahren erkennen, ob ein Tumor gut- oder bösartig ist sowie ob ein Krebspatient gut auf eine Chemo-, Immun- oder Strahlentherapie anspricht. Auch über den Einsatz von modernen Ultraschallverfahren bei bösartigen Schilddrü- senknoten diskutierten die Referenten auf dem Podium. Sie können per Sonografie besonders genau identifiziert werden. Schilddrüsenknoten sind sehr verbreitet jeder zweite Deutsche über 60 Jahre ist davon betroffen. Die meisten Knoten sind gutartig, eine frühzeitige und exakte Unterscheidung ist deshalb lebenswichtig.

„Wichtig war uns, dass beim Dreiländertreffen nicht nur die hochmoderne Medizin auf dem Programm steht, sondern jeder Hausarzt, Allgemeinmediziner und Berufsanfänger hier gleichermaßen die Gelegenheit hat, sich zu informieren und upzudaten“, erklärte Professor Hahn. Zudem wandten sich die Fachgesellschaften in diesem Jahr auch an den ärztlichen Nachwuchs. Neben einer eigens für Studenten ausgerichteten Einführung und Sitzungen zum Thema UItraschallausbildung bot die DEGUM wieder ein studentisches Anwenderseminar mit Theorie- und Praxisteilen an. „Schon im Stu- dium sollten angehende Ärzte Ultraschallverfahren kennenlernen. Diese Möglichkeit geben wir ihnen mit unserem erweiterten Programm“, betonte Dr. Weskott, ebenfalls Kongresspräsident. Die DEGUM ist gerade dabei, ein eigenes Curriculum für Studierende zu entwickeln.

Der Kongress bot den 1700 Teilnehmenden 84 Refresher-Kurse und 12 Anwenderseminare. In 21 wissenschaftlichen Sitzungen und wissenschaftlichen Postern gingen sie auf Themen aus der Grundlagenforschung und der angewandten Ultraschalltechnologie ein. Im Rahmen der praxisorientierten Weiterbildung lernten und übten Ärzte in „Hands-On-Workshops“ diagnostische Techniken mit neuesten Geräten. Begleitet wurde das Programm durch eine Industrieausstellung von pharmazeutischen und medizintechnischen Firmen und Fachverlagen. 